and newspapers have been restricted to a few essential facts. Naturally, they cannot represent a full catalogue. The large international organizations have been mentioned with their complete name as well with their usual abbreviations. Generally only wellknown abbreviations - as indicated in the following pages - have been used.

The trilingual appendix with the graphic diagrams of important organizations and a map of Africa with its new political borders is hoped to contribute to a better optical view of the often confused connections and corelations. The footnotes following the catchwords refer to the corresponding appendix in the respective language.

\title{
Indications rédactionnelles
}

Avant l'emploi du dictionnaire - et pour tirer le meilleur parti de ses possibilités - le lecteur devra observer les indications suivantes:

L'ouvrage contient environ 14000 acceptions françaises, allemandes et anglaises, classées dans le s e ul ordre alphabétique. Des titres en tête de chaque page permettent de trouver plus facilement le mot cherché. Chaque terme (en caractères gras) est suivi d'une définition ou explication aussi brève que possible, limitée au domaine politico-économique. Les auteurs ont renoncé à signaler au lecteur les autres acceptions possibles.

En bas du texte explicatif qui suit le mot se trouve (en caractères italiques) la traduction dans les deux autres langues. Si l'orthographe d'un mot est identique dans deux ou dans les trois langues, le mot en question n'apparaît qu'une seule fois, à savoir dans sa langue d'origine, alors que les mots dans les deux autres langues sont indiqués chacun par un tiret (-). Pour ne pas compliquer l'emploi de l'ouvrage, ce procédé a été rigoureusement observé même dans les cas où le mot allemand, remplacé par un tiret, devait commencer par une majuscule suivant l'orthographe allemande.

Quant aux acceptions qui ne sont d'origine ni française, ni allemande, ni anglaise, les auteurs ont donné la préférence à l'anglais du fait de la diffusion de cette langue. L'ordre des notes dans les 
deux autres langues en bas du texte explicatif résulte simplement de l'ordre alphabétique. Pour les mots allemands, il faut observer

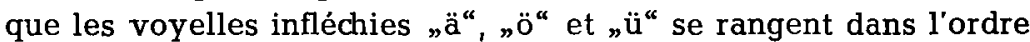
alphabétique sous "ae", „oe" et "ue".

Les flèches $(\rightarrow$ ) dans le texte des définitions renvoient à d'autres acceptions relatives au même sujet et que l'on aura intérêt à consulter pour une meilleure compréhension du terme recherché. L'acception n'est pas répétée dans le texte: elle est remplacée par un tilde ( ) .

Dans les articles concernant les différents pays, l'énumération des partis et des journaux n'est pas exhaustive; elle se limite aux groupes et titres essentiels. Les grandes organisations internationales sont reprises, dans l'ordre alphabétique, aussi bien sous leur appellation complète que sous leur abréviation usuelle. Généralement, les auteurs n'ont employé dans les textes que des abréviations courantes (voir le registre suivant).

L'annexe trilingue, comportant les graphiques des organismes mondiaux importants ainsi qu'une carte de l'Afrique avec les nouvelles frontières politiques sur ce continent, permettra sans doute au lecteur de se retrouver plus facilement dans la complexité des rapports internationaux. Les notes au bas des textes explicatifs renvoient à la page de l'annexe dans la langue correspondante.

\section{Redaktionelle Hinweise}

Für den sinnvollen Gebrauch des vorliegenden Wörterbuchs - und um alle in ihm enthaltenen Möglichkeiten voll ausnutzen zu können - sollte der Benutzer folgendes berücksichtigen:

Das Wörterbuch bringt die rund 14000 Stichworte in deutsch, englisch und französisch in einem integrierten Alphabet.

Das Auffinden der Stichworte wird durch Kolumnentitel am Kopf der Seiten erleichtert. Hinter jedem Stichwort (Fettsatz) folgen die knapp gehaltenen Erläuterungen bzw. Definitionen des Begriffes. Sie wurden thematisch auf den politisch-wirtschaftlichen Bereich begrenzt. Auf Hinweise auf die Bedeutung eines Stichwortes auf anderen Sachgebieten wurde verzichtet. 